\title{
Le phare du Grand Léjon en baie de Saint-Brieuc : une construction en deux étapes (1859-1862/1879-1881)
}

\section{Louis Chauris}

\section{(2) OpenEdition \\ Journals}

Édition électronique

URL : http://journals.openedition.org/abpo/1630

DOI : $10.4000 /$ abpo. 1630

ISBN : 978-2-7535-1485-0

ISSN : 2108-6443

\section{Éditeur}

Presses universitaires de Rennes

\section{Édition imprimée}

Date de publication : 20 mars 2002

Pagination : 107-127

ISBN : 978-2-86847-708-8

ISSN : 0399-0826

\section{Référence électronique}

Louis Chauris, «Le phare du Grand Léjon en baie de Saint-Brieuc : une construction en deux étapes (1859-1862/1879-1881) », Annales de Bretagne et des Pays de l'Ouest [En ligne], 109-1 | 2002, mis en ligne le 20 mars 2005, consulté le 19 avril 2019. URL : http://journals.openedition.org/abpo/1630 ; DOI : $10.4000 / a b p o .1630$ 


\title{
Le phare du Grand Léjon en baie de Saint- Brieuc : une construction en deux étapes $(1859-1862 / 1879-1881)$
}

\author{
Louis CHAURIS \\ Directeur de recherche au CNRS (e.r.) - Université de Brest
}

Les abords et les rives de la baie de Saint-Brieuc - échancrure majeure de la côte septentrionale bretonne - sont jalonnés par une succession de phares : à l'ouest, le Paon et Rosédo à l'île de Bréhat; Porz-Don et Lost-Pic près Paimpol; l'île Harbour au droit de Saint-Quay-Portrieux; Binic; la pointe à l'Aigle au débouché du Légué; à l'est, la Petite Muette au Dahouët; Erquy et Fréhel ${ }^{1}$. Au milieu de la baie, le dangereux récif du Grand Léjon a été signalé en deux temps : d'abord par une tourelle-balise (1859-1862), d'assise assez large pour pouvoir être exhaussée, ultérieurement, en vue de l'établissement d'un feu (1879-1881)². Ce sont les différents épisodes de cette construction, exécutée dans des conditions difficiles, que nous nous proposons de retracer ici $^{3}$. Nous envisagerons successivement le projet primitif et ses modifications, le rôle des ingénieurs des Ponts et Chaussées, le travail pénible des marins et des maçons oeuvrant dans un milieu souvent hostile, les approvisionnements en pierres de construction. Ainsi, peu à peu, sera mieux appréhendé, grâce à cet exemple précis, l'étonnante épopée qu'ont représenté, tout au long du XIX ${ }^{\mathrm{e}}$ siècle, le balisage et l'éclairage des côtes de France ${ }^{4}$, où la pierre des carrières, devenue porteuse de

1. CHAURIS, Louis, "Sur l'emploi des granites du massif de Ploumanac'h-lle Grande dans la construction des phares en Bretagne septentrionale ", Mém. Soc. Emul. Côtes-d'Armor, CXXV, 1996, p. 73-97 et " Les phares de l'île Harbour et de Lost-Pic au large des Côtes d'Armor ", Mém. Soc. Emul. Côtes-d'Armor, CXXVI, 1997, p. 41-52.

2. Plus au sud, le Rohein porte également un feu.

3. À l'aide des documents conservés aux archives départementales des Côtesd'Armor : 11 S 7 (28, 29); S suppléments 561, 579, 580, 581. Nous remercions vivement M.-Y. Le Manac'h, des Affaires maritimes, qui nous a conduit au Grand Léjon.

4. Les publications sur les phares sont innombrables. Nous nous bornons ici à citer deux ouvrages, parus à près de 120 ans d'intervalle : AlLARD, E., Phares et balises, Les Travaux publics de la France, t. V, Paris, Rothschild, 1883, réimpr. Paris, Presses de l’École nationale des Ponts et Chaussées, 1995, 134 p. + 50 planches; Fichou, Jean-Claude, Le Henaff, N. et Mevel, X., Phares. Histoire du balisage et de l'éclairage des côtes de France, Douarnenez, Le Chasse-Marée-Ar Men, 1999, 452 p. 
lumière, atteste encore aujourd'hui, comment l'homme n'a pas hésité à porter sa marque sur les mers (figure 1).

Figure 1 - Le Grand Léjon et les autres phares de la baie de Saint-Brieuc

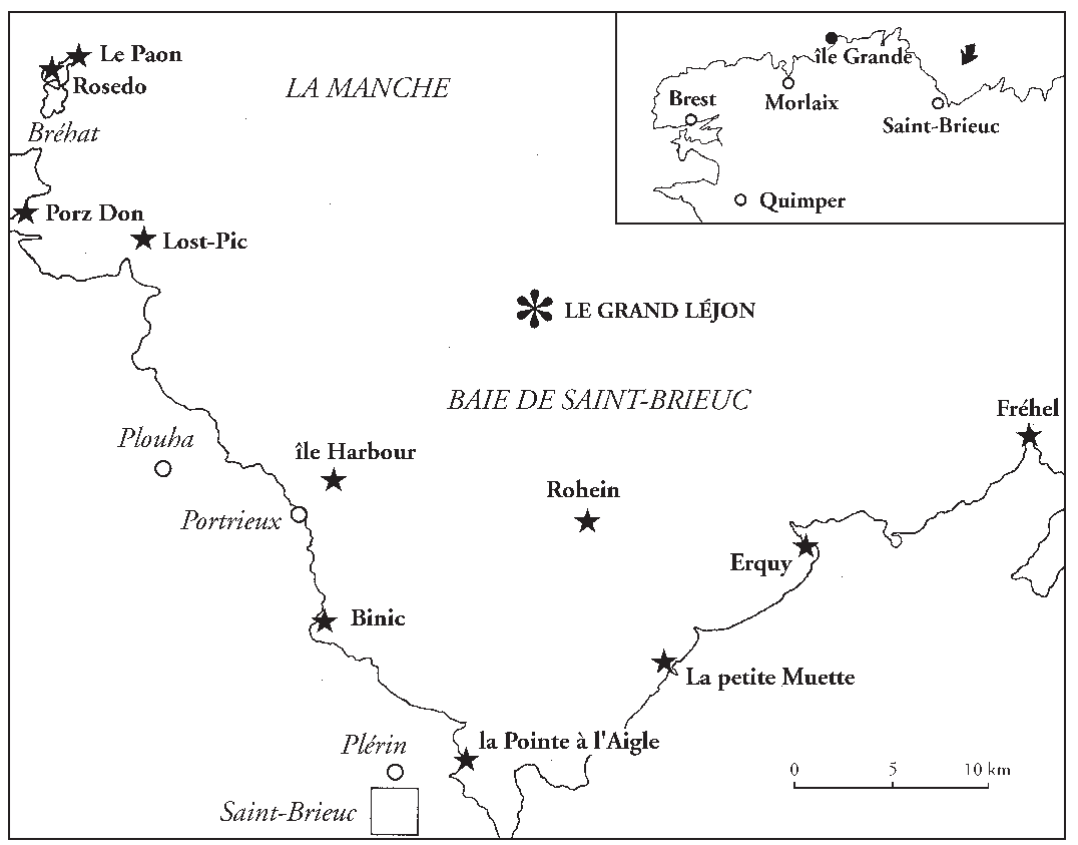

\section{Un platier sauvage isolé}

Figure 2 - Le plateau du Grand Léjon, localisation initialement prévue pour la tourelle-balise (Arch. dép. des Côtesd'Armor, $11 S 7$ [28])

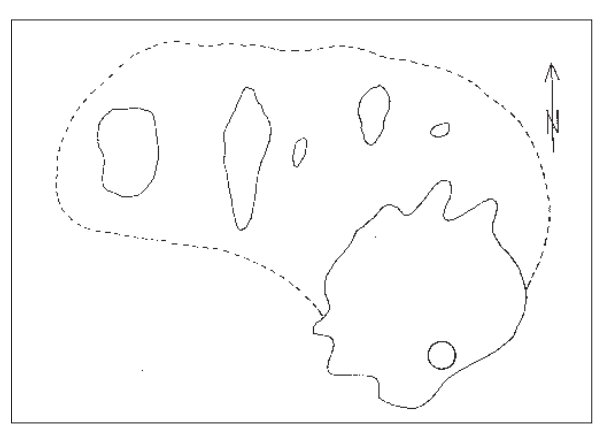

Situé à peu près à égale distance de l'île de Bréhat et du cap Fréhel, sur la route des navires qui se rendent de la Manche dans les ports de la baie de SaintBrieuc, la roche du Grand Léjon est d'autant plus dangereuse que, fort éloignée des côtes ${ }^{5}$, elle s'avère difficile à relever exactement. Elle surgit à proximité immédiate de fonds atteignant localement près de 30 mètres sous le zéro des cartes marines et commence seulement à découvrir à mi-marée. Elle dessine un

5. À environ $16 \mathrm{~km}$ des rivages de Saint-Quay-Portrieux. 


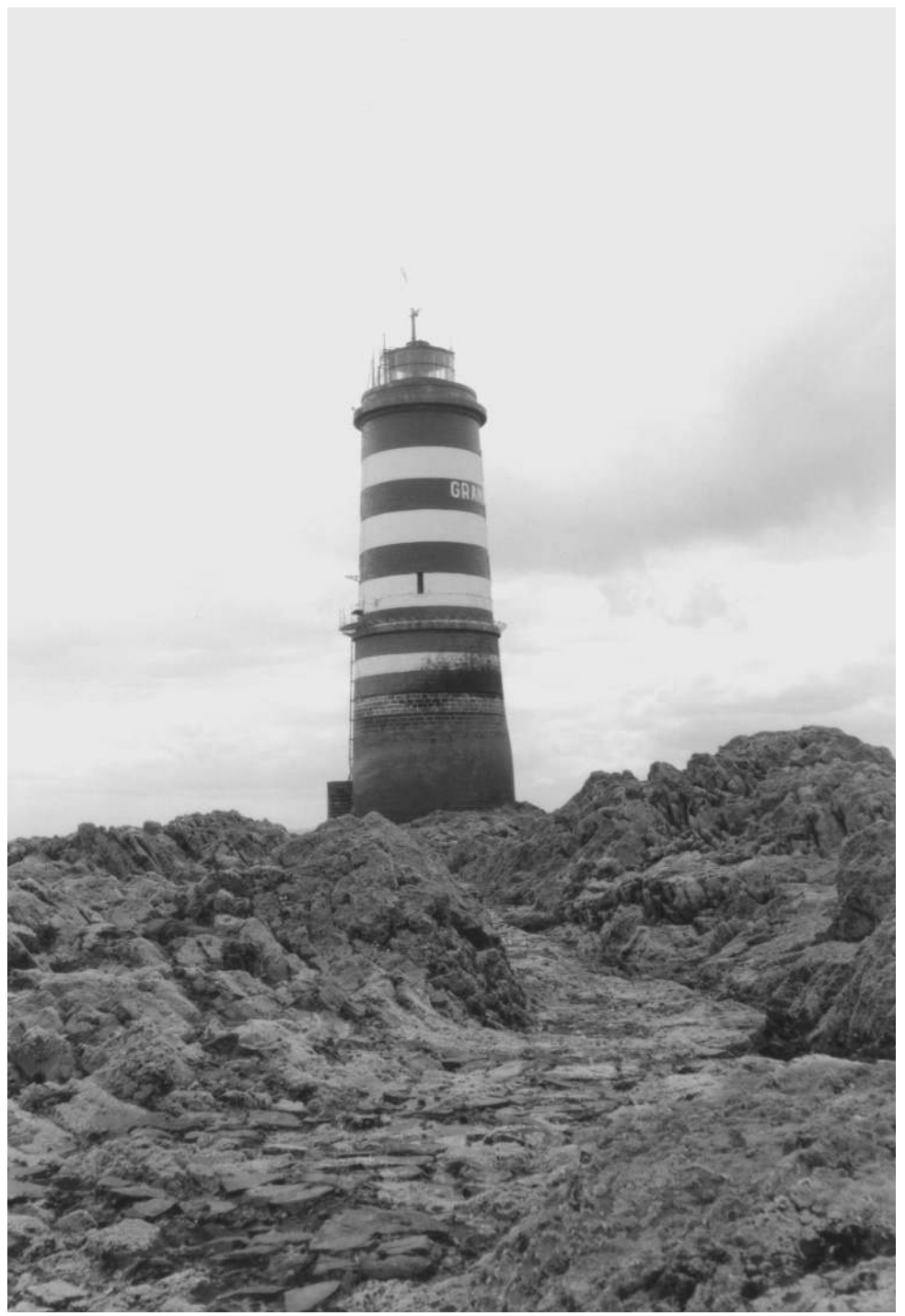

Le platier et le phare du Grand Léjon (Cliché Louis Chauris, 13 août 1999)

Le récif du Grand Léjon forme un platier offrant de spectaculaires affleurements d'une roche volcanique à grain fin, souvent en bancs peu inclinés, beige en surface, gris-noir verdâtre à la cassure. La morphologie et la fraîcheur de la roche attestent la présence de points d'extraction, lors de la construction de l'ouvrage.

Le platier du Grand Léjon est parcouru par plusieurs pistes - entaillées dans le substratum rocheux et empierrées avec la roche locale ainsi qu'avec la diorite de Saint-Quay - permettant l'accès à différents sites d'accostage. 
plateau assez arasé, dépourvu de points hauts et séparé par un étroit chenal d'un groupe de roches beaucoup moins importantes (figure 2). La teinte beige superficielle de la roche et le tapis de balanes qui revêt de larges surfaces, confèrent au platier une coloration claire singulière. En fait, à la cassure, la roche - de nature volcanique, dépourvue de schistosité - offre une nuance sombre, dans les gris-noir verdâtre. Le fond, très fin, est ponctué de minuscules plages de sulfure de fer jaune vif et de petites " taches " vertes. Les affleurements se présentent en bancs de faible puissance, parfois peu inclinés. Les fissures, orientées selon diverses directions, entraînent, sous le choc du marteau, un débitage en fragments irréguliers.

\section{Un premier projet très élaboré}

En 1849, le ministre des Travaux publics demande des renseignements sur le balisage de l'écueil du Grand Léjon. Par une dépêche datée du 31 août 1855, il invite les ingénieurs du département des Côtes-du-Nord à préparer un projet de tourelle en maçonnerie, surmontée d'un mât avec ballon. Selon le rapport de l'ingénieur ordinaire, en date du 18 décembre de la même année, l'ouvrage sera constitué d'une tourelle cylindrique de $4,70 \mathrm{~m}$ de diamètre, d'une hauteur totale de 9,90 m, s'élevant à 3,50 m au-dessus des plus hautes mers de vives-eaux. Le socle, également cylindrique, de 4,90 m de diamètre, doit être complètement noyé dans la roche; le fût, couronné par un pointement conique de $0,20 \mathrm{~m}$ de hauteur. La forme cylindrique a été retenue parce que, à la différence d'une tourelle tronconique, les pierres pourront être taillées, pour l'essentiel, selon deux gabarits seulement. Si la forme cylindrique est " moins élégante qu'une forme légèrement conique, elle se recommande par la simplicité des appareils ". Dans ces conditions, l'exécution ne subira pas de retard en cas de pertes des pierres.

Selon le devis, l'emploi de pierres de taille, posées par carreaux et boutisses, avec une hauteur d'assise comprise entre 0,32 et $0,38 \mathrm{~m}$, est réservé au parement vu. En vue d'assurer la solidité de l'ouvrage, il est spécifié que " toutes les pierres de taille d'une même assise seront reliées et rendues solidaires au moyen de clefs en granit logées dans des rainures verticales pratiquées dans chaque joint, à $0,30 \mathrm{~m}$ de distance du parement ${ }^{6}$ ". Par contre, l'intérieur de la tourelle sera en maçonnerie de blocage granitique. Toutes les maçonneries devront être exécutées à mortier de chaux hydraulique de Saint-Malo. Les pierres de taille en granite seront extraites des bancs les plus durs et les plus sains des carrières de Saint-Brieuc; elles rendront un son clair sous le choc du marteau. Les moellons bruts des maçonneries de remplissage proviendront également des carrières de granite de Saint-Brieuc. Quant au sable, en grains d'un à deux millimètres, il sera prélevé sur la grève de Portrieux.

6. Comme le note l'ingénieur, l'emploi des clefs supplée tout naturellement aux imperfections de la pose; ces prismes, en s'enfonçant, chassent le mortier dont on a eu soin de garnir les rainures, dans les lits et les joints, de manière à remplir les vides involontairement laissés entre les pierres du parement. 
Les exigences sur la qualité du travail sont très strictes. Qu'on en juge! "Toute pierre qui serait écornée ou épaufrée, sera remplacée et ne pourra être remployée qu'après une retaille et dans un emplacement différent où elle conviendrait. " Les modalités de la mise en œuvre sont décrites avec précision. En ce qui concerne le parement vu, " on commencera par présenter la pierre, on la relèvera ensuite, pour la ragréer au besoin, puis on nettoiera et on humectera les surfaces qui doivent être en contact avec le mortier. On étendra alors sur le lit de la pierre et sur le joint de la voisine une couche de mortier de $0,015 \mathrm{~m}$ et on posera sans cale. La pierre sera assujettie et frappée dans tous les sens à coups de masse en bois... La largeur des lits et des joints sera rigoureusement de 0,007 m ». Dans les maçonneries de remplissage, les moellons seront " placés à la main et serrés par glissement les uns contre les autres. Un quart d'heure avant l'emploi, [ils] seront lavés à grande eau sur le tas".

Les prix du granite de Saint-Brieuc, destiné à l'exécution de la tourelle se répartissent comme suit. Pour la pierre de taille, $1 \mathrm{~m}^{3}$ pris sur carrière vaut pour extraction et fente (28 F); le transport au Légué (port de SaintBrieuc), chargement et déchargement compris (7 F), soit $35 \mathrm{~F}$; à cette somme s'ajoutent outils et faux-frais $(1 / 10=3,50 \mathrm{~F})$, soit $38,50 \mathrm{~F}$, puis le bénéfice de l'entrepreneur $(1 / 10=3,85 \mathrm{~F})$. Le prix du $\mathrm{m}^{3}$ s'élève ainsi à 42,35 F. Pour les moellons de blocage, les prix sont respectivement de 1, 4, $0,50,0,55 \mathrm{~F}$, soit $6,05 \mathrm{~F}$ le $\mathrm{m}^{3}$; dans ce dernier cas, on notera que le transport revient quatre fois plus cher que le matériau lui-même pris en carrière. Le détail estimatif de l'ouvrage comprend les éléments suivants :

- les déblais de roches pour les fondations de la tourelle $(56,77 \mathrm{~F})$,

- les maçonneries : blocage de granite $\left(86,55 \mathrm{~m}^{3}\right.$, soit 4738,61 F),

- pierre de taille $\left(83,32 \mathrm{~m}^{3}\right.$, soit $\left.1976,35 \mathrm{~F}\right)$,

- parement de taille courbe ou refouillée $\left(167,01 \mathrm{~m}^{2}\right.$, soit 1766,97 F),

- échelle et balustrade (617,03 F),

- indemnité pour le transport à pied d'œuvre des ouvriers et des matériaux (1200 F).

Soit au total 10355,73 F, auquel s'ajoute une somme à valoir pour les dépenses imprévues (1644,27 F). Ainsi, au 12 décembre 1859, le montant total du projet est estimé à 12000 F. L'accès à la roche était reconnu comme " très difficile "; toutefois, il était fait remarquer que l'existence, au sud-est du récif, d'une paroi accore, permettrait, par beau temps, l'accostage sans danger, de gabares à faible tirant d'eau. Il convenait, par suite, de placer la tourelle le plus près possible de ce point de débarquement obligé. L'exécution était prévue pour comporter 40 jours de travail sur la roche; toutefois, en raison du mauvais temps toujours possible, le nombre de voyages ne pouvait être fixé à moins de 60 .

\section{Un projet soumis à d'importantes modifications}

Une dépêche ministérielle, en date du 19 avril 1856, approuve le projet, sous réserve toutefois de modifications, dont la principale est l'obligation 
d'exécuter en régie la construction, en se bornant à la voie de l'adjudication pour la fourniture des matériaux. Il est également demandé d'examiner le point d'embarquement le plus favorable : le Légué ou Erquy? En fait, comme nous allons le montrer, c'est tout le projet primitif qui allait être bientôt remis en cause, tant en ce qui concerne les modalités de l'exécution que la fourniture des matériaux.

Il est apparu que le transport des pierres du continent au Grand Léjon allait soulever de sérieux problèmes. Tout d'abord, la roche, presque toujours battue par la houle, est entourée, en outre, par de violents courants. Dans un rapport en date du 22 avril 1857, l'ingénieur ordinaire, chargé du projet, rend compte, avec force détail, de ses tentatives pour débarquer. Ainsi, le 26 février de cette année, parti du Légué au moment de la haute mer, il lui a été impossible d'aller au-delà des deux tiers de la distance en ligne droite entre ce port et le Grand Léjon; arrivé à ce point, la marée recommençait à monter : il était inutile de prolonger la traversée. Une autre tentative, à partir du Portrieux, devait se révéler plus efficace. Toutefois, au vu de ses observations, l'ingénieur émet une première conclusion : "il est impossible à un navire à voiles de se rendre [en une marée] au Grand Léjon, soit du Légué, soit du Portrieux ", si on veut aborder pour y effectuer un travail. Au départ d'Erquy, la distance est encore plus grande que du Portrieux. En un mot, "si les vents sont favorables pour accoster - c'està-dire faibles, il est impossible de faire les traversées ". D'où la nécessité d'utiliser un remorqueur à vapeur. Le second problème à résoudre est celui du déchargement des pierres sur la roche. Pour l'ingénieur, il est indispensable que ledit déchargement se fasse à marée haute. Dans ces conditions, il faut que le bateau chargé de pierres puisse arriver à temps au Grand Léjon pour flotter au-dessus du rocher, déposer les matériaux dans un emplacement déterminé, proche de la construction et terminer cette opération avant de s'échouer. Seul Portrieux convient comme point d'embarquement. Non seulement c'est le port le plus proche du Grand Léjon, mais c'est aussi celui pour lequel le courant de flot est le moins défavorable pour se rendre à la roche; le Légué est beaucoup trop loin et le courant est à peu près contraire; quant à Erquy, le courant est tout à fait opposé.

En conséquence, les modalités suivantes sont proposées par l'ingénieur. Un remorqueur traînant à sa suite une gabare de 15 tonneaux environ partira de Portrieux. Sur cette gabare seront chargées les pierres de taille formant le parement et les sacs de sable provenant de la grève de Portrieux, "le meilleur de toute la baie pour la construction". Les pierres et les sacs seront déchargés à marée haute sur le rocher en les laissant glisser à la mer entre des points repérés par des balises. Sur le remorqueur seront placés les ouvriers, les outils et la chaux hydraulique. Le levage des pierres s'effectuera au moyen d'un mât haubané implanté dans la tourelle, en y adaptant une corne analogue à celle employée dans les navires. L'ingénieur ne minimise pas les difficultés prévisibles pouvant influencer la marche des travaux, à savoir la destruction par la mer des ouvrages commencés et l'interruption de leur exécution par suite de l'impossibilité de débarquer 
sur la roche, entraînant ipso facto l'inoccupation des ouvriers embauchés et des navires loués. Il fait preuve toutefois d'un solide optimisme, bien qu'il reconnaisse que " la mer soit [souvent] affreuse sur le Grand Léjon " : le bruit qu'elle fait en s'y brisant " s'entend à terre à près de quatre lieues "! Effectivement, les travaux préliminaires vont tempérer cet optimisme. Comme l'écrit l'ingénieur lui-même (22 avril 1857) :

"Le débarquement des matériaux exigeant la pose de trois balises et de deux petits corps-morts, nous avons dû envoyer des ouvriers préparer les emplacements sur le Grand Léjon. Débarqués sur la roche par beau temps, les ouvriers, venus de Bréhat, ont dû se rembarquer précipitamment et ont été se réfugier au Légué, fuyant devant le temps. Là, ils ont dû séjourner deux jours avant de pouvoir retourner chez eux et $a$ fortiori sur la roche."

Le lieu d'extraction des pierres nécessaires à l'exécution de la tourelle est également modifié. Le granite de Saint-Brieuc est abandonné. Les matériaux de blocage proviendront du Grand Léjon lui-même : il est admis alors que l'extraction de la roche, considérée comme " facile ", fournira " des morceaux assez volumineux, fort lourds et très résistants". Par contre, les pierres de taille proviendront de la carrière de Keraliès dans le district de l'île Grande. Il est indiqué que le granite en provenance de l'île Grande est " d'une taille plus facile et d'un prix un peu moins élevé que celui de SaintBrieuc ». Le devis relatif aux pierres de Keraliès, établi le 6 mars 1857, spécifie que "leur couleur sera uniformément le gris-bleu ". Lesdites pierres sont classées en cinq catégories. L'entrepreneur devra approvisionner 240 morceaux de pierres $n^{\circ} 1 ; 240 n^{\circ} 2 ; 480 n^{\circ} 3 ; 20 n^{\circ} 4$ et $10 n^{\circ} 5$; les fournitures seront payées au morceau. Elles seront transportées à Portrieux par mer et disposées sur le terre-plein du quai près de l'enracinement de la jetée. Ces matériaux devront être approvisionnés en cinq fois entre le $1^{\text {er }}$ juin et le 15 juillet 1857.

\begin{abstract}
"Pour régulariser la réception et éviter les doubles emplois, chaque morceau de pierre présenté portera, sur le parement courbe, une bosse saillante de forme hémisphérique telle qu'il est d'usage d'en laisser dans les carrières de Saint-Brieuc pour les matériaux non encore comptés aux ouvriers. Aussitôt la réception faite, cette bosse sera enlevée par un tailleur de pierre au compte de l'entrepreneur."
\end{abstract}

L'adjudication avait lieu le 18 avril 1857 au profit du Sieur Le Saint JeanMarie (9288,80 F, sans rabais). La dépense de construction en régie étant estimée à 17211,20 F, le montant total de dépenses prévues atteignait alors $26500 \mathrm{~F}$, « bien différent de la première estimation qui était évidemment beaucoup trop faible ".

\title{
L'ajournement des travaux
}

L'inspecteur général, directeur du Service des phares et balises, Léonce Reynaud $^{7}$ répondait le 20 mai 1857, à Dujardin, ingénieur en chef à Saint-

7. Léonce Reynaud (1803-1880) devait se rendre célèbre par la construction (18361840) du phare de premier ordre des Héaux de Bréhat que Michelet rapprochait de « la 
Brieuc, sur le nouveau projet pour le balisage du Grand Léjon : " Je veux d'abord vous féliciter pour la bonne pensée que vous avez eu de vous rendre compte par vous-même des difficultés de l'opération et de votre persistance à la mettre en exécution. " Mais c'était pour ajouter aussitôt : "Je suis effrayé des dangers du système de débarquement auquel vous vous êtes arrêté et peut-être plus encore, car avec beaucoup de prudence, on éviterait ces dangers, des entraves que vous créeraient les enlèvements des pierres de taille par la mer. " Et l'inspecteur général de préciser sa pensée : "Dans les mortes-eaux, on ne pourra pas flotter au-dessus de la roche et dans les grandes marées, les courants sont d'une telle violence qu'ils entraîneront vos pierres pour peu que l'action du vent se joigne à la leur ". Dans ces conditions, quelle solution adopter? Selon le directeur du Service, " ce qu'il y aurait de mieux à faire serait de renoncer à l'emploi de la pierre de taille et de construire entièrement en moellons maçonnés en mortier de Portland de Boulogne et rejointoyés en ciment Parker ". Il ajoute que, d'après l'estimation de l'ingénieur ordinaire, "l'extraction du moellon sur la roche se ferait sans grande difficulté ". Peu après, le 30 juin 1857, une dépêche ministérielle faisait savoir " qu'il y avait lieu d'ajourner les travaux "! La même dépêche demandait aussi d'envisager la substitution de moellon brut hourdi au ciment à la pierre de taille avec chaux hydraulique, en vue d'abaisser le coût de l'ouvrage. Ces instructions ministérielles auraient pu ne soulever que des problèmes mineurs, mais tel n'allait pas être le cas et ce, pour deux raisons.

Suite à l'adjudication pour la fourniture des pierres de taille passée le 18 avril 1857 au profit du sieur Le Saint, adjudication approuvée par le ministère le 30 du même mois, ordre de commencer ladite fourniture avait été donné à l'entrepreneur et une partie des pierres était déjà approvisionnée sur le quai de Portrieux quand était arrivée la dépêche ministérielle. En conséquence, ordre avait été donné à l'entrepreneur de suspendre ses livraisons. À cette date, il y avait sur le quai du Portrieux 72 boutisses, 88 carreaux et 157 clefs; ces pierres d'une valeur de $2939 \mathrm{~F}$, avaient été payées à l'entrepreneur. Mais, comme l'atteste le procès-verbal dressé par l'ingénieur de Morlaix dans l'arrondissement duquel se situe la carrière d'où les matériaux ont été extraits, il se trouvait encore sur carrière 46 carreaux, 62 boutisses et 115 clefs complètement terminés; en outre 4 carreaux et 3 boutisses étaient achevés sauf les refouillements; 24 morceaux bruts étaient préparés pour carreaux, 34 pour boutisses. Aussi, le 6 octobre, le sieur Le Saint fait-il connaître tout le préjudice que lui cause l'arrêt des travaux et demande en conséquence que lui soient soldés les matériaux terminés approvisionnés dans la carrière. L'ingénieur en chef

simplicité sublime d'une gigantesque plante de mer ". " Déjà le phare montait assez haut pour que de toutes parts nous le vissions dominer l'horizon. Ma fortune avait grandi avec lui, et il me semblait que chaque assise qui s'élevait venait assurer notre avenir ", devait écrire le grand ingénieur. Professeur d'architecture à l'école polytechnique, directeur du Service des Phares et Balises (de 1846 à 1878), Léonce Reynaud a laissé sa marque, comme nul autre, sur l'éclairage des côtes de France. 
estime qu'il est juste " d'autoriser l'entrepreneur à transporter et livrer ces derniers matériaux, à cause de leur appareil tout à fait spécial ", qui les rend inaptes à toute autre utilisation. Tout en demandant à être autorisé à utiliser ces pierres de taille pour les premières assises de la tourelle projetée, l'ingénieur en chef suggère, dans le cas contraire, de les réserver " pour quelques unes des constructions de balises en projet dans la région ". Par décision du 7 mai 1858, le ministre des Travaux publics autorisait le paiement des matériaux préparés sur la carrière, et accordait pour cet objet, un crédit de $2436 \mathrm{~F}$, l'adjudicataire étant incité à rendre les matériaux au lieu de réception, c'est-à-dire à Portrieux. La résiliation de l'entreprise de la fourniture des pierres de taille pour la tourelle du Grand Léjon était prononcée, mais les intérêts de l'entrepreneur étaient sauvegardés.

La substitution de moellons à la pierre de taille est également sujette à question. Dans un rapport daté du 9 mars 1858, l'ingénieur ordinaire se montre bien conscient des difficultés soulevées par le débarquement des pierres sur le Grand Léjon, mais c'est pour ajouter aussitôt : "Les gabares qui chargent les pierres de l'île Grande pour les transporter sur toute la côte entrent dans des cavités aussi dangereuses, se posent sur des roches où la tenue n'est pas meilleure. " Par ailleurs, il indique que, dans le nouveau système projeté, où la pierre de taille est supprimée, " il faut d'abord s'assurer de la nature des moellons ". Or l'extraction essayée en plusieurs points du Grand Léjon n'a donné, en général, que « des morceaux de pierres aussi mauvais que possible ${ }^{8}$ ". Aussi, il apparaît évident que le Grand Léjon ne pourra fournir que " des moellons assez petits, feuilletés, remplis de plans de clivage "... Pour l'ingénieur, les moellons extraits sur place auraient pu suffire derrière une maçonnerie en pierres de taille : on ne peut pas " le garantir quand ils se trouveront en parement ". Aussi l'ingénieur propose-t-il, " en définitive, de conserver le mode de construction primitivement adopté ".

Suite au rapport de l'ingénieur ordinaire que nous venons de citer, l'ingénieur en chef expose à son tour, le 23 mars 1858, les divers problèmes soulevés par la construction de la tourelle-balise en développant les thèmes esquissés par son subordonné. Ce document reste des plus intéressants pour appréhender les difficultés rencontrées dans l'érection des tourelles en mer. Après avoir indiqué que, dans le projet, le parement devait être construit en pierres de taille, que des gabares auraient débarquées à haute mer sur le rocher, l'ingénieur en chef rappelle que l'" on a objecté au système proposé les difficultés de déchargement sur la roche " et le risque " de perdre une notable quantité de pierres balayées par la mer ", et, en conséquence, demandé l'érection d'une " tour en tronc de cône, entièrement en moellons bruts ", en provenance du Grand Léjon même. L'ingénieur reconnaît volontiers lui aussi que « l'échouage des pierres de parement est difficile et même dangereux ", mais il précise toutefois que "cette opération est tout à fait dans les habitudes des carriers de l'île Grande et des

8. Annotation en contradiction avec ce qui avait été estimé tout d'abord... 
côtes de la Manche. Sur tous ces points, les gabares viennent s'échouer sur les roches au milieu des blocs qu'elles doivent emporter. On les charge à marée basse, au flot on part chargé 9 . L'opération est même plus dangereuse [qu'elle ne le serait au Grand Léjon], car on ne sait jamais quel sera l'état de la mer quand la gabare en charge viendra à flotter ". Dans le cas du Grand Léjon, au contraire, on n'échouerait pas. L'ingénieur ajoute aussi que l'on peut " attendre beaucoup du courage, de l'habilité et de la prudence " des hommes chargés de ces opérations.

L'ingénieur en chef sait que " le système de construction en blocage avec ciment éviterait sans doute tous les dangers de l'échouage... mais il n'est pas non plus sans inconvénient ". En effet, les matériaux extraits du Grand Léjon sont "fort irréguliers et de petites dimensions " : par suite, ils se prêteront " difficilement à l'exécution d'un parement un peu régulier ".

Autres inconvénients. "la roche étant à hauteur de mi-marée ", ne découvre à peu près que pendant six heures, "mais pour peu que les extractions se fassent sur des parties un peu déprimées, et cela arrivera nécessairement ", le temps d'extraction sera encore réduit. Par ailleurs, la pierre prélevée sur place est souvent enduite "d'une couche grasse qu'il faudra purger " pour qu'elle adhère au ciment. Enfin, les extractions devront être effectuées pendant une marée afin d'être utilisées pour la marée suivante. "Or, si on a pu concevoir des craintes pour l'enlèvement des pierres de taille [par la mer], à plus forte raison [doit-on] le redouter pour des pierrailles."

\section{Le début des travaux}

La construction de la tourelle pleine du Grand Léjon ne devait commencer qu'en 1859. Une lettre du directeur du Service des phares et balises, en date du 27 janvier de cette année, demande à l'ingénieur en chef Dujardin de s'occuper de la formation de l'équipage du bateau à vapeur, destiné au remorquage : "Je n'ai pas besoin d'ajouter [précise-t-il] qu'il sera très essentiel de choisir, surtout pour les fonctions de patron et de premier matelot, des marins encore jeunes, sûrs et très intelligents. " Vers la même période (10 février 1859), l'ingénieur ordinaire propose d'exécuter le parement extérieur de la tourelle, qui servira ultérieurement de soubassement à l'établissement probable d'un phare, " en moellons smillés de petit appareil assez réduit de tête pour éviter la taille d'un parement courbe et assez faible de queue également pour pouvoir être aisément porté par un seul homme".

À l'ingénieur Dujardin qui avait proposé un projet de phare assez monumental, l'inspecteur général L. Reynaud devait répondre, le 18 février, par une lettre qui souligne admirablement les responsabilités, tant financières qu'humaines, des grands serviteurs de l'État:

9. CHAURIS, Louis, "Le transport par mer des granites de l'île Grande (Côtes-d'Armor) ", Mém. Soc. Emul. des Côtes-d'Armor, CXX, 1991, p. 75-90. 
" N'oubliez pas, que nos ressources sont très bornées et que c'est pour nous un devoir des plus impérieux que de les ménager avec la plus grande sollicitude. Il ne s'agit pas seulement des finances de l'État, nous avons à nous préoccuper d'intérêts bien autrement sacrés : de ceux de tant de braves gens qui périssent chaque année sur les dangers que nous n'avons pu encore leur signaler. J'aimerais sans doute à faire des monuments, mais ma conscience s'y oppose. Soyez bien convaincu que vous ferez une bonne action, si en réduisant au strict nécessaire ce que vous allez construire sur le Grand Léjon, vous y économisez de quoi planter une tour sur... La Horaine ${ }^{10} \ldots$ Nous devons nous considérer comme investis d'une noble mission en ce qui est du balisage; on ne saura peut-être pas gré aux ingénieurs des obscurs travaux qu'ils auront si péniblement exécutés, mais ils auront la satisfaction d'avoir rendu avec désintéressement de véritables services à l'humanité. "

Les ingénieurs avaient demandé de pouvoir disposer d'un bugalet ${ }^{11}$, appartenant à la Marine impériale, pour les travaux d'exécution de la tourelle. Le 31 mai 1859, le ministère faisait savoir que ledit bugalet était entièrement prêt et que son commandement avait été confié à un maître de cabotage pris dans le quartier de Saint-Brieuc; l'équipage était composé de douze hommes. Les travaux de construction proprement dits commençaient le 24 juin, époque à laquelle était arrivée la chaloupe à vapeur destinée au remorquage des navires transportant les matériaux. La veille, le bugalet de la Marine était mouillé près du Grand Léjon et affourché sur deux ancres.

Les seuls travaux exécutés antérieurement avaient consisté en la préparation de l'emplacement du mât central destiné à lever les matériaux. Le trou pour recevoir le pied du mât, un trou à section carrée de $0,60 \mathrm{~m}$ de côté, avait été la " cause d'un travail d'une difficulté inouïe, par suite de la dureté de la roche et des dimensions à donner à cette excavation ". L'ingénieur précise que "l'usure a atteint le chiffre de 22 fleurets mis hors de service en une marée de 5 heures et demie... » Le 25 juin, à la marée du matin, le mât, d'une longueur de 17,30 m, avec un diamètre de 0,46 m à la base et de $0,35 \mathrm{~m}$ au sommet, était mis en place. À peine avait-on achevé la plate-forme nécessaire à la confection du mortier et posé quelques pierres à la base de la tourelle, qu'il survenait, le 6 juillet, un violent coup de vent qui forçait le bugalet à se réfugier à Portrieux. Le bâtiment était de retour le 8 ; les travaux reprenaient le 9 et, à partir de ce moment, pendant tout le mois et les premiers jours d'août, ils devaient marcher " dans les circonstances les plus favorables ". À peine quelques marées étaient-elles perdues par suite de l'impossibilité d'accoster à la roche. Par contre, depuis le 4 août, "le succès a été moins grand ". Dans une lettre en date du 29 août 1859, adressée au préfet des Côtes-du-Nord, le ministre des Travaux publics annonçait que le directeur du service des phares venait de lui signaler « le zèle et l'intelligence qu'apportent M.M. les ingénieurs ${ }^{12}$ de la direction des

10. Dangereux récif au large de Bréhat.

11. Petit bâtiment de servitude.

12. M. de La Tribonnière, ingénieur ordinaire et M. Dujardin, ingénieur en chef. 
travaux de la tour du Grand Léjon, travaux sur le succès desquels il est maintenant permis de compter... " Ce résultat est dû aussi en partie au dévouement remarquable du conducteur et des ouvriers chargés sous ses ordres de l'exécution des travaux. En conséquence " une gratification de trois jours de solde serait accordée à chacun des ouvriers et marins ", au nombre d'une quarantaine, attachés aux travaux du Grand Léjon. Les travaux avaient dû être suspendus précipitamment le 29 août et, depuis ce jour, il n'avait plus été " possible de faire quoi que ce soit ». En septembre, de forts coups de vent avaient agité la mer pendant toute une semaine, aussi l'ingénieur désirait-il se rendre sur la roche pour vérifier l'état de la tourelle en construction. Dans son compte rendu (20 septembre), il constate avec plaisir que pas une pierre du parement n'a été enlevée. Par contre, il note que les matériaux déposés au pied de la tourelle ont été " un peu dispersés par la mer, mais [que] tous ont été portés dans des cavités où [il] ne doute pas qu'on les retrouve pour la campagne prochaine".

Dans son rapport de fin de campagne 1859, après le 30 septembre, l'ingénieur précise les modalités d'exécution de la tourelle et l'état d'avancement des travaux. Il indique tout d'abord qu'à part le parement " en moellons smillés de 0,20 à $0,22 \mathrm{~m}$ de hauteur d'assise et de $0,35 \mathrm{~m}$ de longueur de queue moyenne ", tout le massif est exécuté en béton assez gros, dans lequel on introduit à grands coups de masse autant de pierres qu'il est possible, d'abord assez grosses, puis entre ces pierres, des fragments moindres et enfin de petits éléments semblables à ceux avec lesquels est fait le béton : "Toutes ces pierres sont battues jusqu'à ce qu'il soit impossible de les faire pénétrer davantage dans le massif. " Dans le même rapport, l'ingénieur présente les causes multiples de l'augmentation des dépenses. La base de la tourelle a été fondée à un niveau notablement plus bas que prévu, pour se rapprocher de l'accore du plateau vers le sud-ouest. Deux gabares avaient été estimées suffisantes; or, il s'est révélé indispensable d'en avoir trois. L'obligation de nourrir les ouvriers de l'Administration a conduit à leur donner la même nourriture que celle des marins de l'État à bord du bugalet. Le temps de travail journalier des ouvriers a été assez court : 5 heures 1/2 lors des grandes marées; 7 heures pendant les mortes-eaux. Des arrêts de travail imprévus ont été dus à l'état de la mer. La quantité de ciment de Portland a été supérieure aux prévisions... Au total, au cours de la campagne de 1859, $228 \mathrm{~m}^{3}$ de maçonnerie ont été exécutées, mais il reste encore $266 \mathrm{~m}^{3}$ à faire. Les dépenses se sont élevées à 31 463,70 F (parmi lesquelles la fourniture de la pierre (2737,42 F) et sa taille (1348,62 F). L'ingénieur ne cache pas ses craintes pour la campagne de 1860 : il prévoit un renchérissement de la main-d'œuvre « à cause de la présence des grands ateliers du chemin de fer dans le département ${ }^{13}$ ". Il estime alors que la tourelle coûtera $67000 \mathrm{~F}$, au lieu des 25000 prévus antérieurement.

13. Il s'agit de la ligne Paris-Brest. 


\section{L'achèvement de la tourelle pleine}

L'inspecteur général, directeur du Service des phares, avait demandé, le 22 septembre 1859, que le bugalet soit remis à la disposition des ingénieurs à partir du 15 mai de l'année suivante. En 1860, dans les premiers jours de juin, un douloureux événement survenait sur les travaux du Grand Léjon. Un violent coup de vent du sud-ouest que rien n'annonçait, mettait tout à coup les embarcations en danger. Le bugalet de la Marine pouvait se réfugier à Saint-Malo, "non sans avoir éprouvé de grandes avaries ". Mais une chaloupe du pays, qui était louée au mois, a sombré avec deux hommes, les frères Bougeard, qu'il a été impossible d'arracher à la mort. L'embarcation leur appartenait, " ils étaient le soutien de leur mère qui est veuve et qui a encore quatre enfants ". Aussi les ingénieurs proposaient-ils d'allouer à ladite veuve un secours de $300 \mathrm{~F}$ par victime. Une campagne si malheureusement commencée ne pouvait être fructueuse : effectivement, seuls $140 \mathrm{~m}^{3}$ qui coûtèrent $44000 \mathrm{~F}$ (soit environ $310 \mathrm{~F} / \mathrm{m}^{3}$ ) furent exécutés $^{14}$. À la fin de la campagne de 1860, la tourelle était montée à 0,70 m environ en contrebas des plus hautes mers d'équinoxe. Les travaux ne devaient reprendre qu'en 1862; au cours de cette dernière campagne d'exécution de la tourelle, furent effectués $180 \mathrm{~m}^{3}$ de maçonnerie ${ }^{15}$. Le chiffre de la dépense n'a pas été retrouvé, mais on sait qu'en 1861, il avait été évalué à $20000 \mathrm{~F}$ : " On ne doit pas être loin de la vérité en supposant que la tourelle pleine, a coûté $100000 \mathrm{~F}$, soit en moyenne $180 \mathrm{~F}^{\mathrm{N}} \mathrm{m}^{3}$ pour le tout. »

Une quinzaine d'années plus tard, en décembre 1877, avant d'entreprendre l'exécution d'une tour creuse, destinée à porter un feu, sur la tourelle pleine du Grand Léjon, l'ingénieur ordinaire résume son opinion sur ce soubassement. Il rappelle que la construction de ses $550 \mathrm{~m}^{3}$ de maçonnerie avait été laborieuse. Effectuée par des ouvriers campés sur un bugalet stationnaire de la Marine de l'État, elle s'est échelonnée sur trois campagnes (1859-1860-1862). L'ouvrage parementé en moellons smillés de petit échantillon, est essentiellement en béton riche en moellons. Il note qu' « au point de vue architectural, la tourelle du Grand Léjon n'est pas gracieuse; en effet, le fruit est moindre à la base que sur le reste de la hauteur, ce qui produit au tiers de la hauteur environ un ventre extrêmement disgracieux ". Il ajoute toutefois, qu' " au point de vue de la solidité, la tourelle offre de très sérieuses garanties ". Aucune lézarde n'a été constatée dans le parement, malgré les assauts furieux de la mer. Le diamètre exceptionnel donné au couron-

14. Alors qu'en 1859 , le $\mathrm{m}^{3}$ était revenu à environ $130 \mathrm{~F}$.

15. Même les modalités de la paye des ouvriers pouvaient poser des problèmes. Dans un rapport en date du 14 juillet 1862, l'ingénieur ordinaire, après avoir précisé que les travaux sont en cours depuis la deuxième quinzaine de juin, ajoute qu'» il est de la plus grande importance que les ouvriers ne reviennent pas à terre, sauf dans les circonstances commandées par l'état de la mer ». Mais « le procédé de payer leurs salaires par mandats directs exigerait leur présence dans une caisse publique ". Or, ne remettre les sommes gagnées qu'à la fin de la campagne "pourrait mettre les familles dans la gêne ". Nous n'avons pu savoir quelle solution avait été adoptée à ce sujet... 
nement (7,40 m) qui s'élève à 3,50 m au-dessus des plus hautes mers, constitue ainsi une base excellente pour l'exécution d'une tour creuse.

\section{La construction du phare}

Sur l'avis de la commission des Phares, une décision ministérielle, en date du 13 juin 1877, avait décidé qu'un feu de $4^{\mathrm{e}}$ ordre serait établi sur la tourelle du Grand Léjon. Suite à la consultation d'une commission nautique, il avait été finalement résolu qu'" on laisserait dans l'obscurité toute la côte d'Erquy et de Dahouët, qu'un faisceau de lumière rouge couvrirait les îles Saint-Quay et que le feu serait blanc sur tout le reste de l'horizon ". Les ingénieurs des Ponts et Chaussées des Côtes-du-Nord (Pelaud et Jourjon) dressent alors le projet d'une tour creuse destinée à porter ledit feu à 16,10 m au-dessus des plus hautes mers d'équinoxe. Selon le devis, en date du 13 décembre 1877, le diamètre externe de la tour creuse est réduit à 7 mètres : cette disposition " permettra de rompre les lignes peu harmonieuses de la base ". Pour encastrer les maçonneries nouvelles dans les anciennes, il est prévu de démolir les trois assises supérieures de l'ancienne tourelle, soit environ $0,75 \mathrm{~m}$. Le fruit extérieur est de 0,04 /mètre, ce qui donne aux maçonneries des épaisseurs voisines de 1,50 m à la base, à $0,82 \mathrm{~m}$ en couronnement. L'ingénieur ordinaire rappelle à ce sujet que « le Grand Léjon reçoit dans les tempêtes d'énormes rouleaux de houle. La vie des deux gardiens dépendra de la solidité de la tour ". L'accès à la porte d'entrée du phare, à établir entre le sud et le sud-est, se fera par une échelle en bronze, de 27 échelons, à poser tout entière sur la maçonnerie de la tourelle. Toujours selon le devis de fin 1877, les matériaux de construction proviendront respectivement de la carrière de Carhuel (massif de Saint-Quay, moellons bruts), des carrières du Pré-Doré (au Légué, moellons d'appareil et pierres de taille); le sable prélevé sur les grèves de Portrieux, sera préalablement dessalé. Un dallage en marbre est prévu dans la chambre de service qui renfermera l'huile minérale.

Le devis et le cahier des charges précités devaient être rectifiés le 15 février 1879, suite aux instructions de la dépêche ministérielle du 22 mars 1878. Ce nouveau document offre un ensemble de données sur la provenance et la mise en œuvre des matériaux. La carrière désignée pour la fourniture des moellons bruts et des quartiers est une carrière "située au sud du moulin de Carhuel " anciennement exploitée pour les perrés de Portrieux; la pierre est granitique ${ }^{16}$ " de couleur bleue et verdâtre ". Les plus gros moellons auront les dimensions suivantes : longueur $(0,40 \mathrm{~m})$, largeur $(0,25 \mathrm{~m})$, hauteur $(0,30 \mathrm{~m})$; les plus petits respectivement $(0,20-$ $0,15-0,10 \mathrm{~m})$. Moellons bruts et quartiers seront livrés à Binic, lieu d'embarquement. Les carrières désignées pour les moellons schisteux dégrossis sont celles du Pré-Doré ou des côtes de Plérin. "À une assise de moellons d'appareil granitique correspondront, au gré de l'entrepreneur, une, deux ou trois rangées de moellons dégrossis. "Pour les pierres de taille et

16. En fait, il ne s'agit pas d'un granite, au sens des géologues, mais d'une diorite. 
les moellons d'appareil granitiques, les carrières désignées sont celles de Saint-Brieuc; toutefois, " on admettra la substitution du granite de l'île Grande à celui prévu, mais à condition que les encadrements de toutes les baies se correspondent et que les moellons smillés d'une même assise en parement vu aient la même provenance".

De même que pour la construction de la tourelle, il paraissait indispensable de mouiller le chantier du phare sur un bugalet stationnaire de la Marine de l'État. Ledit bugalet servirait à la fois de logement pour les hommes et de magasin pour les matériaux qu'on ne peut laisser au contact de l'eau salée. Le personnel - y compris l'équipage du navire - ne devant pas dépasser 50 hommes, il était demandé 50 hamacs garnis. Le bâtiment souhaité devrait jauger de 180 à 200 tonneaux. Un différent devait opposer le ministère de la Marine au Service des Ponts et Chaussées au sujet du commandement du bugalet. Le ministre de la Marine avait prescrit d'en donner commandement à un maître de timonerie de la Marine de l'État. Mais les ingénieurs des Ponts et Chaussées considéraient cette mesure comme préjudiciable à la bonne marche des travaux : "Ce n'est pas le capitaine du bugalet qui commandera les ouvriers et fera construire les maçonneries, mais bien un de nos employés. " Après avoir indiqué qu'ils avaient déjà fait le choix d'un maître au cabotage, les Ponts et Chaussées développent leur manière de voir : "Comment un tel chantier peut-il marcher si notre employé et le capitaine ne s'arrangent pas? " Et d'ajouter : "Notre employé est un ancien marin; depuis 20 ans, il surveille le balisage de tout le littoral du département. C'est peut-être le meilleur pilote de nos parages. " Autre argument : la mesure prévue est " préjudiciable à la sécurité de l'équipage et aussi des ouvriers campés sur le bugalet. À dix milles des côtes, au milieu d'écueils, le navire mouillé devra fuir dès que la position ne sera plus tenable, et il pourra être obligé de lâcher ses corps-morts la nuit. Quelle confiance peut, dans de pareilles conditions, inspirer un maître en timonerie qui ne connaît ni les parages, ni les rochers, ni les courants, ni les feux? " D'où l'avis de prier le ministre des Travaux publics de bien vouloir s'entretenir de ce problème avec son collègue de la Marine...

Les travaux de construction du phare, estimés à $90000 \mathrm{~F}$, débutaient en 1879. Le bugalet, "Solide ", venant de Cherbourg, servait de logement aux ouvriers. Les gabares assuraient l'acheminement des matériaux depuis Binic, ainsi que le servie de la poste et des vivres au Légué. Le conducteur des travaux sur le rocher, M. Le Bozec, sous les ordres de l'ingénieur Jourjon, avait cru pouvoir se passer des services du vapeur "Confiance". Cette idée devait s'avérer " malheureuse ", par suite des périodes de calme : la première campagne fut très lente et seul le rez-de-chaussée fut élevé. Il allait en être tout autrement l'année suivante : "L'ingénieur Guillemoto, qui venait de prendre le service, mit à la tête du chantier $\mathrm{M}$. le conducteur Le Renard. " Ce dernier commença la campagne " par l'installation d'un échafaudage très ingénieux, autour du rez-de-chaussée déjà construit ". Son plancher, formant une plate-forme de 2,50 m de large autour de l'ouvrage 
en construction, était à l'abri des plus grosses lames... En dépit d'un déradage de plusieurs jours, dû à un violent coup de vent d'ouest, la campagne de 1880 devait être " exceptionnellement active ", puisque tout le gros œuvre était achevé à la fin de septembre. Les escaliers en fonte et l'appareil optique étaient ensuite mis en place; le feu était allumé le 20 juin 1881.

Quelques problèmes, d'ordre divers, s'étaient posés lors de la construction du phare. L'un d'eux concerne la réclamation, par la Marine militaire, d'une somme élevée - près de $4000 \mathrm{~F}^{17}$ - pour la location du bugalet mis à la disposition des ingénieurs en 1879, " alors qu'on avait pensé que ce bugalet, nécessaire pour avoir une assez grande quantité de matériaux et tenir en dehors des heures de travail les ouvriers à proximité de la tour, serait prêté gratuitement ". Aussi, en 1880, les ingénieurs renvoyaient-ils ce bugalet et se procuraient un bateau de commerce... à des conditions beaucoup moins onéreuses ${ }^{18}$. Par ailleurs, en 1879, la chaloupe qui servirait de transbordement des matériaux du bugalet à la tour avait été perdue et il avait fallu la remplacer par une nouvelle, au prix de 2248 F. Notons aussi que, par lettre du 29 juillet 1880, des meuniers, à Ponto et au Moulin de la Grève ${ }^{19}$ se plaignaient d'" une installation faite dans le ruisseau pour le lavage du sable destiné à la construction du phare ". Enfin, l'entrepreneur, en raison des facilités d'extraction avait demandé à employer, au lieu du granite de Saint-Brieuc, qui nécessitait un long transport par terre, le granite de l'île Grande, offrant, en échange, le transport par mer gratis jusqu'à Binic. Dans ces conditions, il suffisait d'effectuer un charroi de $2 \mathrm{~km}$ par terre, aux frais de l'Administration. De là, une économie réalisée sur le transport de la pierre.

Les dispositions du phare sont les suivantes. Le rez-de-chaussée, servant de magasin à l'eau et aux huiles, a un diamètre intérieur de 4 mètres. Au premier étage, où se situe la cuisine, le diamètre est de 4,30 m; au deuxième étage, le diamètre de la chambre à coucher des deux gardiens est de 4,50 m; la chambre de service, au troisième étage, offre un diamètre semblable. L'accès du rocher au phare, s'effectue par une échelle en bronze, scellée dans la paroi de la tourelle pleine. La hauteur de la tour creuse est de 12,40 m depuis le dallage de l'entrée jusqu'au sommet du parapet; le plan focal se trouve à $23 \mathrm{~m}$ au-dessus du rocher, c'est-à-dire à la cote 28,40 m par rapport au zéro des plus basses mers et à 16,20 m au-dessus des plus hautes mers. Par décision ministérielle du 22 mars 1881, le service du Grand Léjon entraînait la création de trois emplois nouveaux de gardiens, respectivement de deuxième, troisième et quatrième classe. À l'achèvement complet des travaux du phare, les dépenses faites se répartissaient de la manière suivante : à l'entreprise pour fourniture de matériaux seulement $(11832,19 \mathrm{~F})$, en régie $(107160,45 \mathrm{~F})$, soit 118992,60 F.

17. Exactement $3885 \mathrm{~F}$.

18. La location de la goélette qui a servi de casernement pendant trois mois en 1880, est revenue à $2700 \mathrm{~F}$.

19. Entre Portrieux et Étables. 


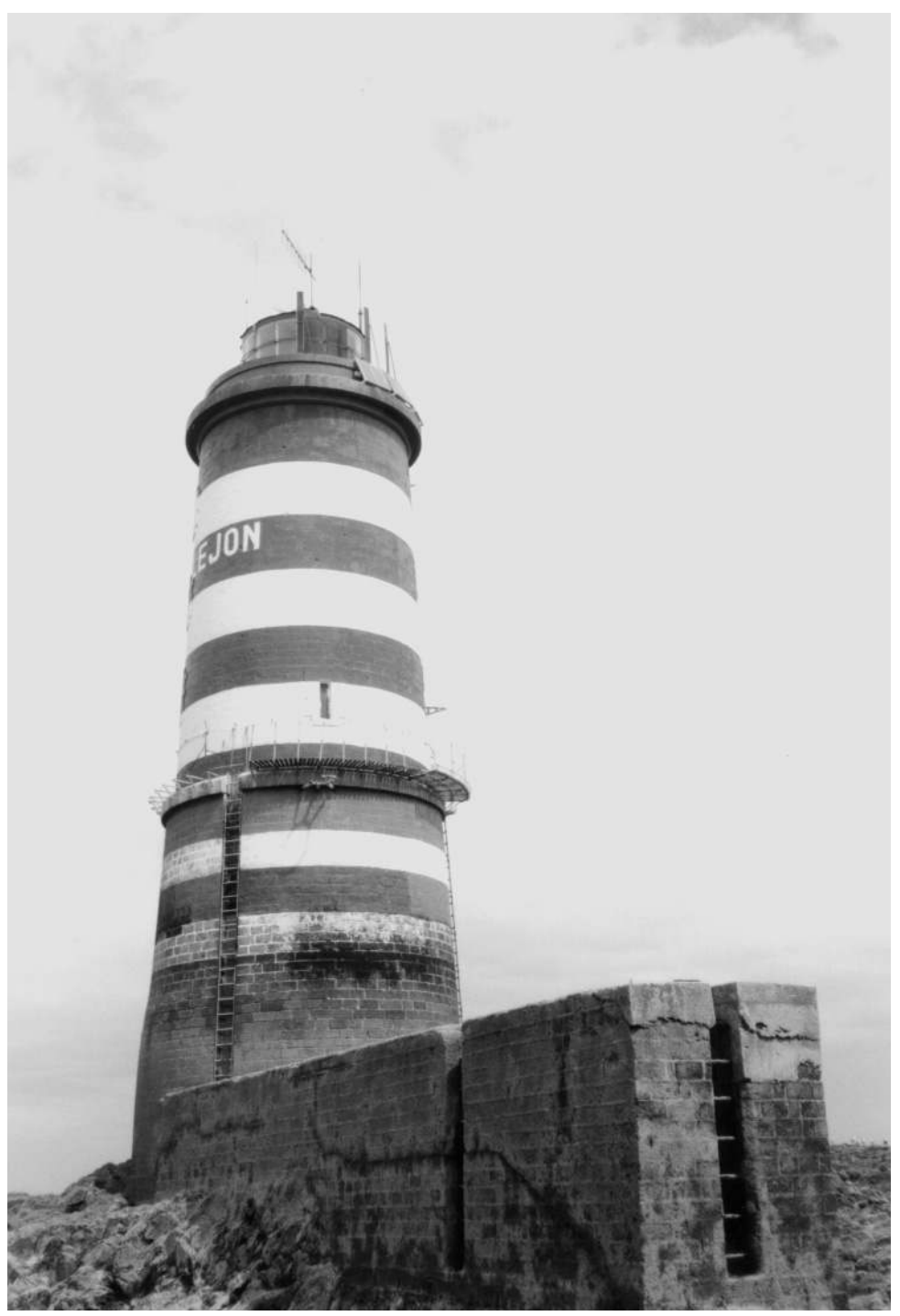

Vue d'ensemble du phare du Grand Léjon (Cliché Louis Chauris, 13 août 1999)

Au premier plan, cale plus tardive, avec parement en parpaing. La partie inférieure de l'ouvrage - jusqu'au haut de l'échelle - en maçonnerie pleine, correspond à la tourelle-balise (1859-1862); la partie supérieure, creuse, coïncide avec le phare proprement dit (1879-1881). La peinture, en bandes alternantes rouges et blanches, masque le plus souvent la pierre. 
L'augmentation des dépenses ayant été justifiée, le ministre ordonnait l'ouverture du crédit demandé par les ingénieurs.

\section{L'amélioration des conditions d'accostage et d'accès}

Figure 3 - Amélioration des conditions d'accostage au Grand Léjon proposées en 1890, la différence des contours du récif entre les figures 2 et 3 est fonction des hauteurs de la marée (Arch. dép. des Côtes-d'Armor S supplément 581)

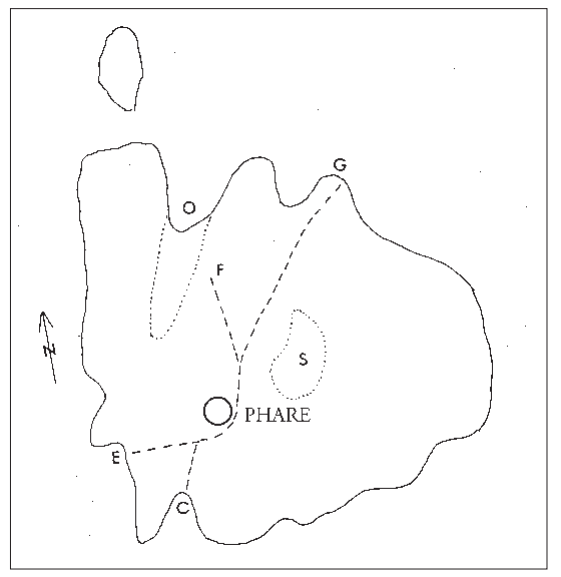

Dans un rapport en date du 6 septembre 1890, l'ingénieur ordinaire déplore qu'il n'existe alors aucun ouvrage servant à l'accostage au Grand Léjon. Il précise que " les débarquements se font très péniblement, à marée basse sur un rocher abrupt et que les opérations sont loin d'être exemptes de danger pour le personnel et pour le matériel ». La roche comporte trois petites anses où l'on peut débarquer à marée basse selon les modalités suivantes (figure 3). Avec les vents du nord, l'accostage se fait dans le sud-ouest en $\mathrm{C}$ ou en E selon la houle et la hauteur de la mer; par vent d'ouest, dans le nord-est en G. Pour faciliter les cheminements des divers accès jusqu'au phare, l'ingénieur suggère d'effectuer quelques dérasements de rochers et de combler les trous et

les failles. Il propose en outre la construction d'une petite cale de débarcadère au sud-ouest, à la suite du dressement $\mathrm{BC}$, déjà exécuté depuis plusieurs années. Cette cale, de 1,20 m de largeur, une pente de 0,13 m par mètre sur une longueur de 6 mètres, permettrait un débarquement facile et sans danger à marée basse. Ces travaux seraient exécutés avec des moellons en provenance de l'île de Bréhat, déposés dans une coulée du rocher (S) où la mer n'a qu'une action très faible. Les gardiens du phare euxmêmes effectueraient le dressement du rocher et les maçonneries. L'ensemble du travail reviendrait à $1600 \mathrm{~F}$.

D'autres améliorations devaient être également proposées. Lors de la construction de la tourelle pleine, une échelle avait été posée dans une rainure pratiquée sur le côté ouest; mais la porte du phare ayant été placée dans le sud-est, il avait été nécessaire d'implanter une autre échelle, en saillie, au pied de ladite porte. D'où l'avis d'établir une petite galerie en fer entourant le phare entre les deux échelles, à la hauteur du seuil de la porte : il sera alors possible d'accéder à l'entrée par l'une ou l'autre de ces échelles, selon la direction des vents. Dernière amélioration : établir également par le bas une communication facile entre les deux échelles au moyen d'une espèce de risberme, avec plate-forme d'un mètre. Ces modifications ont 
été réalisées à une date que nous n'avons pu encore préciser. On remarquera toutefois que la petite cale prévue primitivement s'est transformée en un ouvrage imposant et qu'aux moellons de Bréhat ont fait place des parpaings.

Au terme d'une analyse détaillée relative à la construction d'un phare en mer, envisagé dans son lent déroulement événementiel, il apparaît nécessaire de dégager, au sein de cette trame historique complexe, tout en soulignant les particularités présentées par le Grand Léjon, quelques aspects de portée plus générale sur les travaux publics maritimes entrepris en Bretagne au XIX ${ }^{\mathrm{e}}$ siècle.

1- La primauté de l'intérêt général. Suite aux travaux de Fresnel ${ }^{20}$, il devenait possible d'envisager un programme d'ensemble pour l'éclairage des côtes de France. En Bretagne où se situent les atterrages les plus dangereux du pays, cette gigantesque entreprise devait s'échelonner sur près d'une centaine d'années ${ }^{21}$. L'objectif était de contribuer ainsi à sauver le maximum de vies humaines. Dans le cas du Grand Léjon, cet aspect devait être remarquablement exposé par le directeur du service des phares, Léonce Reynaud. On notera toutefois que l'allumage de ce feu a été relativement tardif (1881), nettement postérieur à celui de Fréhel (1847), où il existait déjà un feu dès la fin du XVII ${ }^{\mathrm{e}}$ siècle, mais antérieur à celui de Lost-Pic $(1894)^{22}$.

2- L'obligation de procéder par étapes. Les impératifs budgétaires ne permettaient pas toujours une construction en une seule fois. Le Grand Léjon est très représentatif de cet état des choses, puisque l'exécution du phare proprement dit a été précédée, plus d'une quinzaine d'années auparavant, par l'érection d'une tourelle-balise. Mais même au cours des diverses étapes, l'exécution restait soumise aux conditions météorologiques : impossibilité de travailler pendant la mauvaise saison et parfois, dans la campagne d'été, suspension des travaux pendant quelques jours.

3- Le polylithisme des matériaux de construction. L'exemple du Grand Léjon est très symptomatique de la diversité dans la nature et l'origine des pierres utilisées. Un tel polylithisme reflète, au moins en partie, les modifications successives apportées au projet. Primitivement, les parements vus de la tourelle-balise pleine devaient être élevés, selon un appareillage particulièrement soigné, avec un granite relativement distal (Saint-Brieuc); le même granite devait aussi fournir les moellons de blocage. En fait, lors de l'exécution des travaux, les changements dans la provenance des pierres vont s'avérer assez spectaculaires, avec appel à la roche volcanique extraite sur place, la diorite de Saint-Quay, relativement proche, le lointain granite de l'île Grande...

20. Entraînant une amélioration très considérable dans l'éclairage des phares.

21. Saint-Matthieu (1835), Kereon (1916). Plusieurs phares, détruits par les Allemands en 1944, ont été reconstruits après la dernière guerre.

22. L'allumage de l'île Harbour remonte à 1850, de Binic à 1854, de la pointe à l'Aigle à 1857, de Rosédo et du Paon (à Bréhat) à 1860. 
4- L'organisation complexe du chantier. À l'inverse du phare des Pierres Noires dans le Léon ${ }^{23}$ où le chantier de base était installé dans une grande île (Beniguet), voisine de la tour à ériger, au Grand Léjon, les ouvriers étaient logés dans un navire (bugalet, puis goélette) mouillé à proximité de la roche. Une telle manière d'opérer était liée à l'impossibilité de ramener la main-d'œuvre journellement à terre, vu la situation de l'ouvrage loin au large. Il reste aujourd'hui à se représenter l'activité déployée : extraction dans les carrières; acheminement par charrois ou par mer des pierres jusqu'à Binic; transport desdites pierres, par gabare, de ce port au Grand Léjon; débarquement souvent délicat; maçons sur l'échafaudage, élevant la tour; équipage du navire de servitude, prêt à embarquer les ouvriers en cas de mauvais temps inopiné, tous à la merci d'un accident, ainsi qu'en témoigne la mort de deux marins en 1860; souci constant de l'ingénieur et du conducteur des travaux... Plus que tout autre ouvrage, l'érection d'un phare en mer reflète les efforts persévérants d'une équipe solidaire.

5- L'impact financier. L'augmentation très considérable des dépenses initialement prévues pour les travaux au Grand Léjon se doit d'être soulignée. À l'évidence, le projet primitif avait nettement sous-estimé les difficultés de l'ouvrage, dues aux aléas d'une exécution en pleine mer et, par suite, son coût réel. Les $12000 \mathrm{~F}$ initialement prévus pour la tourelle pleine devaient être largement dépassés, puisque les ingénieurs estimaient que ce seul ouvrage avait dû revenir à une somme de l'ordre de 100000 F!

6- L'architecture. À l'inverse de nombreux phares qui sont de remarquables chefs-d'œuvre ${ }^{24}$, le Grand Léjon, comme le reconnaissaient les ingénieurs eux-mêmes, n'est pas un beau monument, en particulier à cause du " ventre " présenté par la tourelle du soubassement. Une des raisons du manque de " pureté " des lignes est, sans doute, à rechercher dans la modification du fruit de la tourelle pleine, simplement parementée en moellons. On notera toutefois l'effort des ingénieurs pour corriger, lors de la construction de la tour creuse, ce que ce soubassement pouvait avoir d'inesthétique. Mais, ultérieurement, l'exécution de la massive cale, parementée en parpaings, en vue de faciliter les conditions d'accostage, le " corsetage " local de la partie inférieure de la tourelle et les escaliers permettant d'accéder à l'enracinement basal des échelles ont, quelque peu, à leur tour, défiguré l'aspect de l'ouvrage ${ }^{25}$.

23. Pris, parmi bien d'autres, en exemple. Voir à ce sujet CHAURIS, Louis, "Construction d'un phare en mer d'Iroise : les Pierres Noires (1866/1872) ", Annales de Bretagne et des Pays de l'Ouest, 105, 1998, 1, p. 71-89.

24. Comment ne pas penser ici aux Héaux de Bréhat, à Eckmülh, à l'île Vierge...

25. Le phare du Grand Léjon est aujourd'hui automatisé. Le secteur blanc a une portée de 18 milles; le secteur rouge, de 14 milles. Tour ronde, tronconique, à larges bandes alternatives rouges et blanches, l'ouvrage est aisément identifiable. Mais ces peintures rendent difficiles les déterminations pétrographiques in situ des matériaux employés. À l'évidence, la roche en place a été largement utilisée pour les moellons de blocage de la partie inférieure, pleine, ainsi que l'atteste la multiplicité des points d'extraction, qui permettent, encore aujourd'hui, d'obtenir des échantillons très sains. Les moellons de 


\section{RESUME}

Les péripéties présentées par l'exécution, en deux périodes (18591862/1879-1881), du phare du Grand Léjon en baie de Saint-Brieuc, soulignent les difficultés rencontrées lors de l'éclairage au large des côtes bretonnes. Modifications successives des projets, changements dans la nature et la provenance des matériaux de construction, hardiesse des ingénieurs, des ouvriers et des marins œuvrant avec persévérance dans un milieu souvent hostile, fortes augmentations des dépenses prévues... autant de facettes qui, grâce aux données d'archives, éclairent, à l'aide d'un exemple précis, l'histoire trop peu connue des grands travaux publics maritimes en France dans la seconde partie du XIX ${ }^{\mathrm{e}}$ siècle.

\section{ABSTRACT}

The incidents presented by the construction, in two stages (1859-1862/18791881), of the Grand Lejon lighthouse in the Saint-Brieuc bay, underline the difficulties met during the lighting off the coast of Brittany. The successive modifications of the projects, the change in the nature and origin of the stones, the boldness of the engineers, workmen and sailors, working with perseverance in rather hostile surroundings, high increases of the expenses originally planned, as many facets which, owing to archives, throw a light, just by one precise example, on the misunderstood history of the great maritime public works undertaken in France in the second half of the XIXth century.

parement, en assises régulières, de la tourelle, semblent bien avoir été façonnés dans la sombre diorite de Saint-Quay-Portrieux - en fait, le matériau de qualité le plus rapproché du Grand Léjon. Par contre, les parties observables dans la tour creuse - comme l'entourage de la porte d'entrée - ont été taillées dans l'un des granites de l'île Grande. On retrouve ainsi au Grand Léjon l'emploi de la pierre de ce district, si recherchée pour les phares de la région (voir note 1). Les petites pistes qui sillonnent le platier rocheux pour conduire à divers points d'accostage, ont été empierrées avec la roche locale, mais aussi, fort curieusement, avec la diorite de Saint-Quay et même avec des fragments schisteux bleu-noir, en provenance du continent. 
